\title{
Une approximation de la prévision saisonnière des étiages et sécheresses en Catalogne
}

\section{An approximation to seasonal forecasting of low flows and droughts in Catalonia}

\author{
LLASAT, M.C., A. ZARAGOZA \\ Groupe d'Analyse de Situations Météorologiques Adverses (GAMA). Département d'Astronomie et Météorologie. \\ Université de Barcelone. \\ Av. Diagonal 647, 08028 Barcelone. Espagne. \\ Tél : +34 9340211 24, Fax : +34 9340211 33, e-mail : carmell@am.ub.es
}

J. CABOT, B. AZNAR

CLABSA. Barcelone, Espagne.

$L$

es étiages et les sécheresses sont une caractéristique hydro-climatique en Espagne. La dernière sécheresse qui a affecté l'Espagne a été extraordinairement grave en Catalogne, où elle s'est prolongée entre l'année 2004 et le printemps de 2008. L'objectif de cette contribution est de montrer l'évolution de cette dernière sécheresse dans le cadre de la caractérisation des sécheresses en Espagne, ainsi qu'une approximation pour faire une prévision saisonnière du débit des rivières qui nourrissent les principaux barrages des Bassins Internes de la Catalogne et la ville de Barcelone. Etant donné que les principales rivières naissent dans les Pyrénées et PréPyrénées, nous avons centré l'analyse pluviométrique sur cette région. En premier lieu, nous avons caractérisé les situations météorologiques associées au déficit de précipitation en la région d'étude. Ensuite, nous avons créé des séries de précipitation mensuelle représentatives des trois bassins d'étude, en utilisant 127 stations pour la période 1940-2008 et nous avons obtenu la distribution par centiles, valeurs qui seront utilisées pour calibrer le modèle hydrologique et obtenir le rang des débits prévus. Nous avons analysé toutes les prévisions disponibles sur des sites Internet et, après une validation pour la période 1998-2008 nous avons sélectionné les prévisions du modèle ECM4. Alors, nous avons créé un index de prévision saisonnière comme combinaison linéaire des valeurs climatiques et la prédiction de ECM4, qui a aussi été validée. Cette information a été introduite dans un modèle hydrologique et nous avons recréé l'évolution prévue de la dernière sécheresse, en considérant les demandes potentielles de la population. Nous avons trouvé une considérable amélioration sur la prévision du volume d'eau retenu aux barrages, amélioration qui pourra être appliquée dans le futur. L'avantage de cette méthode est qu'on peut l'appliquer sur la base de prévisions saisonnières de libre diffusion. Ce travail a été développé dans le contexte du projet SOSTAQUA.

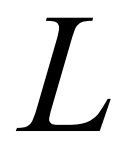

ow flows and droughts are a hydro-climatic feature in Spain. The last drought that has affected Spain has last four years in Catalonia from 2004 to the spring of 2008. The aim of this contribution is to show its evolution and the potential use of seasonal forecasting to improve the water management in Catalonia. Due to the fact that the principal rivers in the Internal Basins of Catalonia born in Pyrenees and Pre-Pyrenees region the analysis of the precipitation is focused on this region. Monthly precipitation series representatives of the three basins of study have been obtained from 127 raingauges for the period 1940-2008. These series have been used to calibrate the hydrological model and to validate the seasonal forecasting. All seasonal predictions from internet sites have been analysed and one have been selected ECM4 and later validated for the region of study with 127 raingauges for the period 1998-2008. Then a seasonal prediction index has been created as a linear combination of climatic data and the ECM4 prediction that has been validated too. This information has implemented into a hydrological model and it has been applied to the last drought considering the real water demands of population. It has been found a considerable advance in the forecasting of water volume into reservoirs that can be applied in the future. The advantage of this methodology is that it only requires seasonal forecasting free through internet. This work has been developed into SOSTAQUA project. 


\section{I — INTRODUCTION}

Les sécheresses constituent un problème typique en Europe [Lloyd-Hughes et Saunders, 2002] et Espagne depuis des temps historiques et elles ont même été la cause de mouvements migratoires. L'existence de sources documentaires et la pratique de rogations "pro pluvia" [Martín Vide y Barriendos, 1995] permet d'identifier et de caractériser de graves périodes de sécheresse historique, comme la période 18121818, qui a affecté le Littoral Méditerranéen et les Baléares [Álvarez et al, 2008] ou encore les sécheresses enregistrées en Catalogne dans la période connue comme Oscillation Maldà [Barriendos et Llasat, 2003], qui ont provoqué la perte de la majorité des récoltes en Catalogne. C'est pour cette raison qu'on a décidé, vers le milieu du $\mathrm{XX}^{\mathrm{e}}$ siècle, de construire de nombreux barrages qui permettraient de réguler l'approvisionnement en eau de l'agriculture et des villes. L'administration hydraulique a dû et doit encore traiter des problèmes générés par les sécheresses en Espagne [MIMAM, 2000], que ce soit en situation d'urgence comme cela a été le cas de la dernière sécheresse [MIMAM, 2007] ou encore dans le cadre de la planification hydrologique. Dans un cas comme dans l'autre, la gestion des barrages est cruciale. Etant donné l'existence de Communautés Autonomes, cette gestion peut ou bien dépendre de l'Etat central, ou bien de la Communauté Autonome elle-même. C'est le cas des Bassins Internes de la Catalogne, dont l'organisme responsable est l'Agence Catalane de l'Eau ("Agencia Catalana del Agua"), de la "Generalitat de Catalunya".

Lors de la période 2004-2007, une sécheresse a eu lieu en Espagne, plus particulièrement intense entre 2004 et 2005, et qui, dans le cas des Bassins Internes de Catalogne, s'est prolongée jusqu'à mai 2008, mois où elle a brusquement été interrompue par une période de pluies intenses et étendues. Cette sécheresse a été associée à un déficit en pluies généralisé dans toute la Péninsule, malgré certaines périodes ponctuelles de fortes tempêtes et même d'inondations comme celle de septembre 2006 [Barnolas et al, 2008]. En Catalogne, les barrages sont arrivés à enregistrer des valeurs au-dessous de $15 \%$ de leur capacité et le Décret de Sécheresse promulgué par la Generalitat a été prolongé jusqu'à décembre 2008. A la différence de cette période commune à toute l'Espagne, on peut relever d'autres cas comme la période 1992-1995, qui a principalement affecté les bassins du sud de l'Espagne, mais pas la Catalogne. Cette différence en extension provient surtout de la distribution du déficit de précipitations, et par conséquent, des différentes situations météorologiques. Par exemple, lors des années 1983-1986 et 1997-2001, on a enregistré un déficit pluviométrique généralisé, et même plus intense que l'épisode récent dans certains points. L'impact a cependant été moins important que dans la période 2004-2007, à cause de l'augmentation de la consommation d'eau enregistrée ces dernières années.

Le cycle hydrologique lui-même et la gestion des ressources comportent un décalage entre les causes météorologiques responsables du déficit de précipitation et la sécheresse. Cependant, la caractérisation météorologique est fondamentale pour une meilleure compréhension des futures prévisions. Dans le cas des Pyrénées, région qui fait l'objet de ce travail, le déficit pluviométrique est dû à la présence d'ano- malies positives mensuelles qui altèrent le champ de géopotentiel, de sorte que les anticyclones se déplacent vers des régions inhabituelles pour cette période de l'année [Olcina, 2001 ; Zaragoza, 2009]. Lors des sécheresses d'hiver, cette anomalie est encore plus prononcée et située sur le nordouest de la Péninsule. Au printemps et en automne, on enregistre des valeurs anormales de géopotentiel généralisées sur tout le territoire européen ayant pour centre le golfe de Gascogne et la Bretagne française, respectivement, même si elles n'arrivent pas à être aussi intenses qu'en hiver. En été, les variations en relation avec le champ moyen sont beaucoup plus petites que ce soit en intensité ou en dimensions, et un dipôle apparaît avec des valeurs faibles sur l'Atlantique nord et une hausse dans le centre de l'Europe. La compréhension de ces facteurs est importante pour l'analyse des résultats des prédictions saisonnières qui sera présenté ci-après.

\section{DONNÉES}

\section{II.1 DONNÉES PLUVIOMÉTRIQUES}

La Catalogne dispose de trois réseaux principaux fournissant des informations pluviométriques : le réseau d'AEMET (Agence d'Etat de Météorologie - Agencia Estatal de Meteorología) qui couvre toute la Catalogne et possède des données mensuelles de 1152 pluviomètres depuis 1940, malgré une certaine discontinuité ; le réseau SAIH (Système Automatique d'Information Hydrologique - Sistema Automático de Información Hidrológica) de l'Agence Catalane de l'Eau, qui dispose de 126 stations avec des relevés toutes les 5 minutes pour les Bassins Internes de Catalogne (Cuencas Internas de Cataluña - CIC) depuis 1996 ; le réseau XEMA (Réseau de Stations Météorologiques Automatiques - Xarxa d'Estacions Meteorològiques Automàtiques) du Service Météorologique de la Catalogne, avec 43 stations dans la moitié nord de la Catalogne et avec des relevés horaires depuis 1995.

Cette contribution se centre sur les hauts bassins des rivières Cardener, Llobregat et Ter, qui alimentent respectivement les barrages la Llosa-Sant Ponç, la Baells et de Sau-Susqueda. Le domaine choisi, que nous désignerons désormais par "domaine TLL" (Fig. 1), est bien représenté d'un point de vue pluviométrique à partir des données de 127 stations ( 85 de l'AEMET, 26 du SAIH et 16 du XEMA), qui sont celles qui ont été utilisées, que ce soit pour la période 19402000 pour le calibrage hydrologique et l'obtention de centiles ou pour la validation effectuée pour la période 1998-2008.

\section{II.2 MODÈLE SAISONNIER : ECM4}

Après avoir évalué la qualité et la fiabilité de diverses prédictions saisonnières de libre accès dans la région étudiée, le modèle ECM4 a été choisi car il présente des informations à partir du mois de mai 1998 et jusqu'à présent, même si la période 1998-2000, étant une période d'essais, ne sera pas utilisée dans ce travail. Le modèle ECM4 prédit la pluie trimestrielle jusqu'à 6 mois plus tard au moyen de cinq sorties trimestrielles $(\mathrm{M}+0, \mathrm{M}+1, \mathrm{M}+2, \mathrm{M}+3$ et $\mathrm{M}+4$ où $\mathrm{M}$ est le mois de départ et $\mathrm{M}+\mathrm{i}$ est le premier mois du 


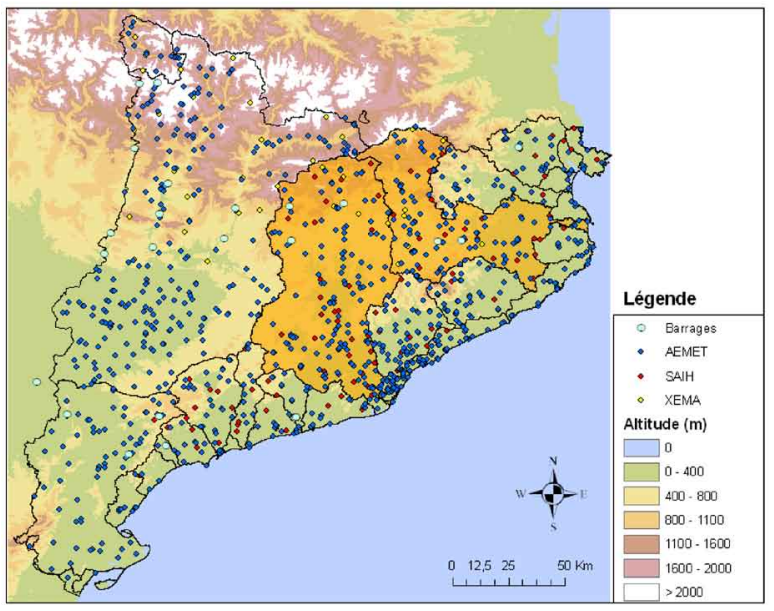

Figure 1. Domaine d'étude à la Catalogne, avec les trois réseaux pluviométriques, les barrages et les deux bassins principaux étudiés.

trimestre objet de prédiction). Le modèle fournit la probabilité selon laquelle on peut enregistrer chacune des catégories suivantes : AN (above normal), $\mathrm{NN}$ (near normal) o BN (below normal). Sur la base de ces informations, un tableau des prédictions a été élaboré en lisant le graphique pour les pixels situés dans la zone TLL. Vu la résolution graphique de la sortie du modèle, l'erreur associée à chaque lecture est de $\pm 5 \%$, en prenant la valeur moyenne de chaque intervalle de la légende.

\section{III — METHODOLOGIE}

\section{III.1 PRÉCIPITATION}

La taille du domaine TLL est comparable à celle du pixel utilisé pour le modèle ECM4, raison pour laquelle on utilisera une seule série de pluie mensuelle représentative de toute la région et correspondant à son barycentre géographique. La série mensuelle du barycentre est obtenue comme moyenne pondérée par la distance entre chaque pluviomètre et le barycentre, selon la formule suivante (1)

$$
p_{\text {cent }}(t)=\sum_{0}^{M} \frac{p_{i}(t)}{d_{i}^{2}}
$$

où $p_{\text {cent }}(t)$ est la pluie accumulée dans le barycentre et au mois $t$, calculée à partir des $M$ pluviomètres disponibles avec des registres de pluie $p_{i}(t)$ pour ce même mois $t$, et se trouvant chacun à chacun à une distance $d_{i}$ du barycentre. Nous travaillons avec une moyenne mobile trimestrielle avec des intervalles de 1 mois, et pour l'obtenir les terciles qui permettront d'ajuster les seuils de précipitations correspondants aux trois intervalles prévus par le modèle ECM4 et de valider le modèle combiné de prédiction saisonnière. Pour l'application postérieure au modèle hydrologique, on procède de la même manière mais en obtenant les séries correspondan- tes aux trois barycentres représentatifs des bassins étudiés et pour lesquels les prédictions seront réalisées.

\section{III.2 MODÈLE COMBINÉ}

Pour obtenir le modèle combiné de prédiction saisonnière, on applique le procédé proposé par Fraedrich \& Smith [1989] et Fraedrich \& Leslie [1987], basé sur une combinaison linéaire entre deux prédictions probabilistes : celle du modèle ECM4, et la prédiction climatologique. Cette dernière donne une prédiction équiprobable $(33 \%)$ pour les trois terciles de pluie trimestrielle.

La combinaison linéaire optimale $(\varphi)$ est celle qui obtient une erreur quadratique moyenne (MSE) minimale, est elle est obtenue avec la formule (2), où $\langle\cdot\rangle$ est une moyenne temporelle, $\varphi_{1}$ et $\varphi_{2}$ sont le modèle ECM4 et le modèle climatique, et $\delta$ est la série de pluie trimestrielle du barycentre TLL.

$\phi=a \phi_{1}-b \phi_{2} \quad a=\frac{\left\langle\delta \phi_{1}\right\rangle-\left\langle\delta \phi_{2}\right\rangle+\left\langle\phi_{2}^{2}\right\rangle-\left\langle\phi_{1} \phi_{2}\right\rangle}{\left\langle\phi_{1}^{2}\right\rangle+\left\langle\phi_{2}^{2}\right\rangle-2\left\langle\phi_{1} \phi_{2}\right\rangle} \quad b=1-a$

On obtient 15 coefficients distinct, un pour chaque tercile et pour chaque horizon, au moyen des séries $\varphi_{1}, \varphi_{2}$ et $\delta$ de la période août 2001-décembre 2005 ("période de calibrage"), et à partir de ces derniers on calcule la valeur du modèle combiné pour toute la période complète, depuis août 2001 jusqu'à décembre 2008. Le calcul étant effectué avec la probabilité du modèle ECM4, le résultat du modèle combiné présente aussi une erreur associée (E) selon la formule (3).

$$
\varepsilon_{\phi}=a \phi_{E C M 4}
$$

\section{III.3 VALIDATION}

On utilise le BPS (Brier Probability Score) comme indicateur de la justesse des prédictions catégoriques, tout comme l'appliquent Gibergans \& Llasat [2007]. Le BPS est compris entre 0 et 2 , le BPS $=0$ étant la prédiction parfaite. On calcule de même le degré de justesse des catégories prévues (réussites et fausses alarmes) au moyen d'un tableau de réussites et d'erreurs, à ne pas confondre avec le tableau de contingence. Les deux validations sont réalisées pour les trois modèles : ECM4, modèle climatique et modèle combiné, et pour deux périodes distinctes : d'une part, pour toute la période de prédictions disponible (août 2001 - octobre 2008), désormais "période totale", et de l'autre pour la période qui n'a pas été utilisée dans le calibrage (janvier 2006 - octobre 2008), désormais "période opérationnelle".

\section{III.4 APPLICATION MÉTÉOROLOGIQUE AU MODÈLE HYDROLOGIQUE}

Pour l'application de ces prédictions au modèle hydrologique utilisé, à chaque sortie du modèle correspondent les trois probabilités associées à chaque catégorie ( $\mathrm{AN}, \mathrm{NN}$ et $\mathrm{BN})$ délimitée par les valeurs minimales et maximales de pluie 
définies par les terciles trimestriels. De tels seuils de pluie sont mensuellement désagrégés au moyen des mesures pour les barycentres correspondant aux trois groupes de barrages, et on attribue à chaque mois la même probabilité que celle présentée par le trimestre en question.

\section{III.5 ÉVOLUTION DU VOLUME D'EAU RETENU PAR LES BARRAGES}

Dans l'actualité, la méthodologie pour estimer l'évolution $\mathrm{du}$ volume d'eau retenu par les barrages est mise en œuvre en considérant en premier lieu l'état réel des réserves. En deuxième lieu, on considère les apports mensuels d'eau qui correspondraient aux barrages pour lesquels on souhaite faire la prédiction, selon les séries historiques d'apports enregistrés [ACA, 2000]. En troisième lieu, on soustrait pour chaque barrage la demande attendue pour le mois en question (Fig. 2).

Cette méthodologie fournit des informations sur la probabilité selon laquelle on disposera d'un volume déterminé de ressources stockées dans un futur proche, même si l'estimation de cette probabilité est fondée sur la probabilité d'occurrence des apports historiques associée à l'histogramme observé, et non pas sur la situation météorologique concrète du moment où la prédiction est effectuée. Dans ce travail, les prédictions météorologiques saisonnières ont été employées pour modifier la probabilité des apports prévisibles dans les prochains mois, ce qui a ainsi permis d'obtenir une nouvelle estimation des volumes de réserve associés aux nouvelles probabilités d'occurrence selon la prédiction météorologique.

Dans ce travail, l'évolution des ressources retenues par les barrages a été estimée en réalisant deux simplifications méthodologiques nécessaires pour simplifier le calcul, en attendant que l'intérêt soit centré sur l'évaluation de la prévision météorologique. D'une part, comme modèle de transformation de pluie-débit à l'entrée du barrage, on a utilisé un coefficient d'eau de ruissellement mensuel obtenu à partir des données réelles de la période d'étude juillet 2006 - juillet 2008. D'autre part, comme demande attendue, on a utilisé la donnée réelle de débit mensuel évacué du barrage dans chaque système, au lieu de supposer une demande théorique. Ceci permet d'éliminer de la méthodologie l'incertitude liée à l'évolution de la demande qui est par ailleurs beaucoup plus réduite que celle des apports.

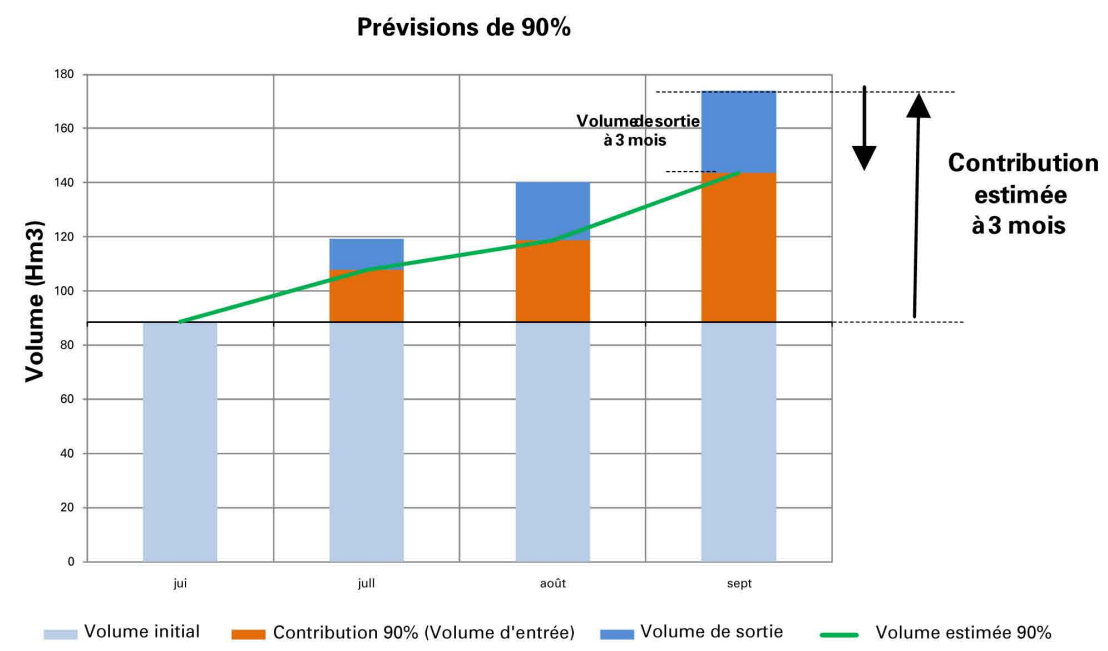

Figure 2 : Exemple d'évolution accumulée des apports et du volume dans les barrages pour les trois mois suivants. On considère le centile $90 \%$ pour les apports.

\section{IV — RÉSULTATS}

\section{IV.1 MODÈLE COMBINÉ}

Dans le calcul du modèle combiné selon (2), on obtient les indices $a$ et $b$ (Fig. 3). Ces indices sont représentés au moyen d'un graphique en barres pour observer les différents poids attribués par la combinaison à chacun des deux modèles.

En se basant sur les valeurs des coefficients ou poids, on a pu constater que la combinaison est fondée en grande partie sur le modèle ECM4 pour le tercile central, sur la prédiction

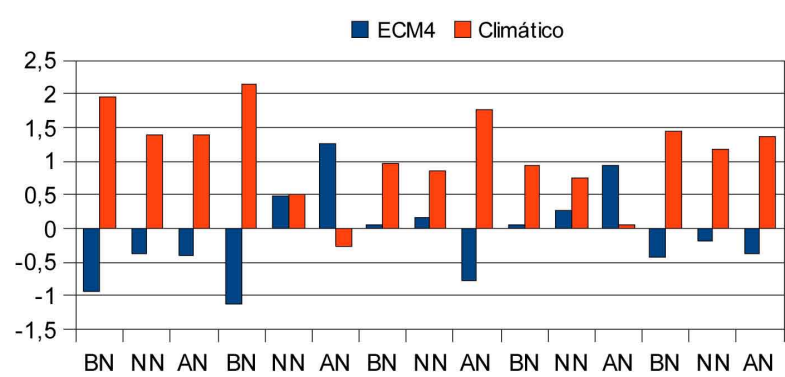

Figure 3 : Coefficients du modèle combiné, pour tous les horizons et terciles (août 2001 - décembre 2005) 
climatique pour le tercile supérieur et que selon l'horizon de prédiction, les poids varient aussi sensiblement pour chaque modèle. Ces observations permettent de remarquer que le modèle ECM4 présente une bonne qualité prédictive pour le tercile $\mathrm{NN}$, alors qu'il est un mauvais prédicteur pour le tercile supérieur, probablement lié à la composante locale élevée de pluies intenses en Catalogne.

Selon la formule (3), l'incertitude est plus grande dans les cas où la combinaison accorde un plus grand poids au modèle ECM4 comme conséquence du processus de propagation d'erreurs, de sorte que lorsque la capacité de prédiction de la combinaison augmente, l'incertitude qui y est associée augmente aussi, et vice versa. Par conséquent, le modèle combiné donne des probabilités qui sont dans une certaine mesure représentatives d'un certain rang, autour de $10 \%$ comme ce qui se produit avec le modèle ECM4.

\section{IV.2 VALIDATION DE LA COMBINAISON}

La Figure 4 montre que le modèle combiné fournit la meilleure prédiction saisonnière pour toute la période et pour les différents horizons de prédiction, la prédiction pour le trimestre $\mathrm{M}+0$ étant la plus favorisée. En outre, cette prédiction est plus stable que celle du modèle ECM4, qui présente des valeurs de BPS dispersées pour tous les horizons. On dénombre les réussites et les erreurs des trois modèles, en

a)
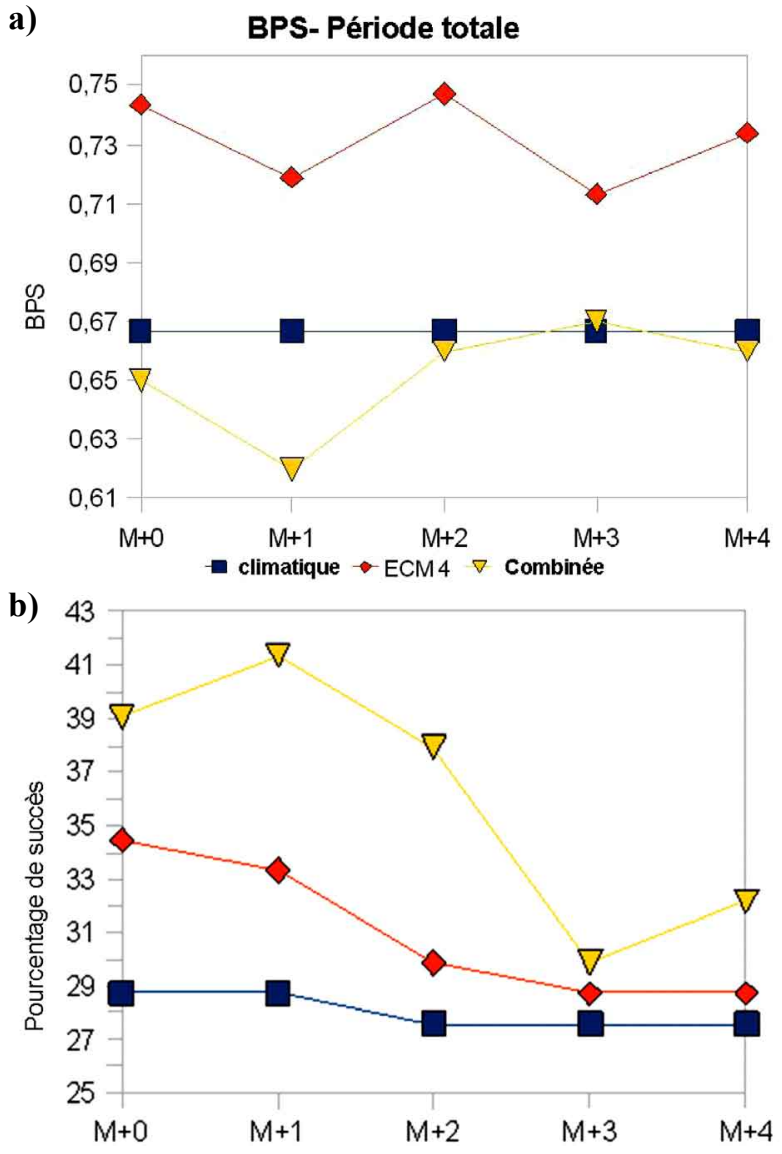

Figure 4 : BPS (a) et nombre de réussites (b) des trois modèles, pour tous les horizons et pour la période totale comparant le tercile prédit avec la plus grande probabilité avec la pluie observationnelle, et, dans les cas où apparaissent des terciles présentant la même probabilité, on donne priorité au tercile le plus petit. Le modèle combiné est la prédiction saisonnière la plus proche de la réalité, étant celui qui présente la proportion la plus élevée de réussites.

\section{IV.3 PRÉVISION DE L'ÉVOLUTION DES RÉSERVES}

A partir des prédictions saisonnières adaptées à la région des barrages étudiés, on réalise une première transformation linéaire au moyen de laquelle on obtient l'apport pour le barrage, avec une probabilité d'occurrence égale à la probabilité offerte par la prédiction de pluie. Comme nous l'avons mentionné plus haut, l'objectif est de voir si l'utilisation de prévisions saisonnières peut améliorer la prévision de réserves calculée à partir de l'information climatique. Pour cette raison, nous présentons les résultats de la prévision de l'évolution des barrages utilisant les prévisions saisonnières ainsi que les résultats de l'évolution ayant l'information climatique pour référence.

Dans la Figure 5, on montre à titre d'exemple l'application de la méthodologie pour obtenir la prévision à trois mois pour le barrage de La Baells. La ligne qui représente l'évolution du barrage est associée à un nombre de 0 à 100 qui est le centile statistique de la prévision. Si le nombre est 5 , cela indique que la possibilité que l'évolution soit plus basse est inférieure à $5 \%$ (très peu probable) ; alors que le nombre 90 indique que la probabilité d'être sous la ligne est très haute. Dans le graphique, on observe en pointillés la prévision $(\mathrm{M}+0)$ en utilisant les centiles climatiques de 90,50 et $5 \%$. Dans les franges colorées, on observe la prévision du volume du barrage obtenue à partir des données de prédictions météorologiques saisonnière combinée pour les différents terciles mensuels. En outre, dans la légende, on indique la distribution de probabilités associées à chaque tercile. Finalement, la ligne noire indique l'évolution réelle du volume du barrage.

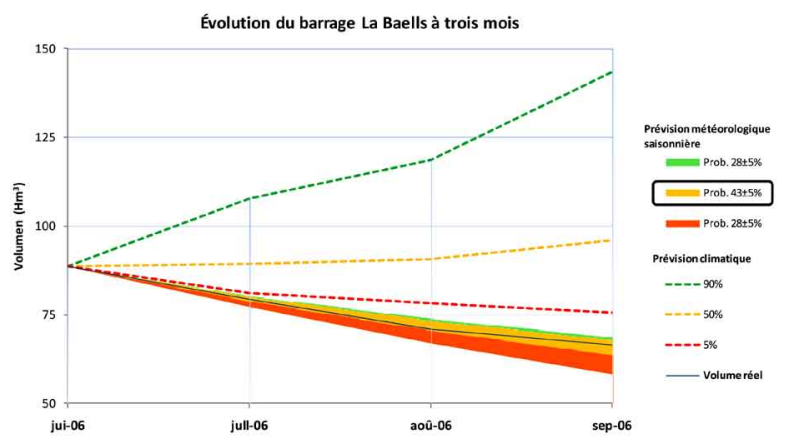

Figure 5 : Exemple d'évolution du barrage de La Baells avec la sortie (juin-juillet-août) commencée en juin $(\mathbf{M}+\mathbf{0})$

L'application de la méthodologie pour toute la période d'étude permet de composer des enveloppes de l'évolution 
pour un, deux et trois mois. La figure 6 montre comment on obtient les enveloppes de un, deux et trois mois avec P90\% (il y a $90 \%$ de probabilité que l'évolution soit plus basse).

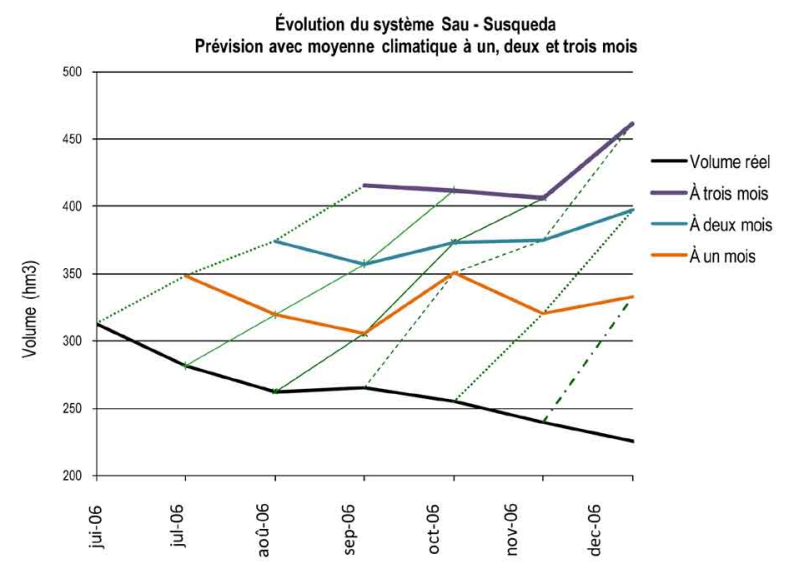

Figure 6 : Prévision avec une moyenne climatique du Système Sau-Susqueda. Enveloppes de $90 \%$ pour un, deux et trois mois

La figure 7 présente l'évolution du volume d'eau dans le barrage de La Baells en appliquant la prévision à trois mois qui est la plus intéressante du point de vue du gestionnaire de ressources. Si l'on observe ce graphique, on peut voir que l'utilisation de prédictions saisonnières permet d'améliorer considérablement le rang de prévision respect la prévision climatique. En outre, on observe que la plupart du temps, l'évolution du volume réel est située entre la frange de $5 \%$ et la frange de $90 \%$ de la prédiction saisonnière (partie en gris), alors que si l'on utilise la moyenne climatique, le volume réel est à de nombreuses occasions au-dessous de $5 \%$. Ceci est dû au fait que les modèles de prédiction saisonnière utilisent la situation météorologique dans laquelle ils se trouvent pour déterminer l'état futur, et qu'ils permettent donc de mieux caractériser les périodes de sécheresse. Par contre, la prédiction climatique considère ces périodes comme très peux probables.

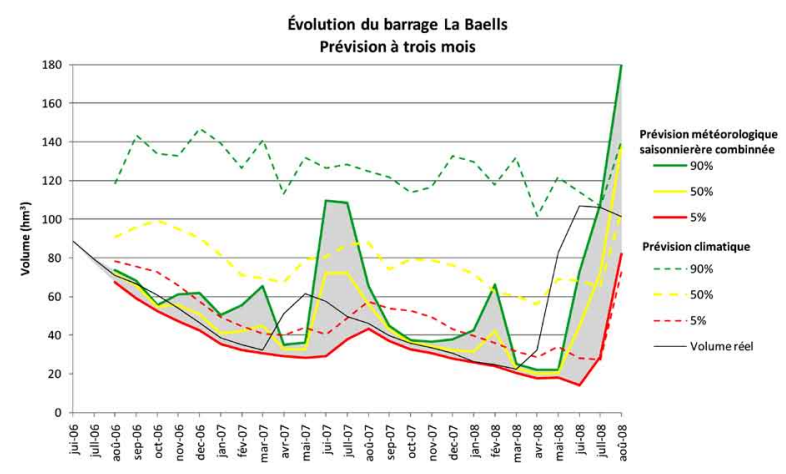

Figure 7 : Prévisions $(\mathbf{M}+0)$ sur trois mois agrégées pour toute la période d'étude utilisée (juillet 2006 - juin 2008).
D'autre part, on observe que la prédiction saisonnière ne pronostique pas toujours de manière adéquate les changements de tendance, comme cela a été le cas des mois d'avril 2007 et d'avril 2008, lorsque s'est produit une augmentation de précipitations suite à des épisodes de Levant (avril 2007), des épisodes convectives très fortes (mai 2007) et des flux marqués du sud-ouest favorisés par des dépressions atlantiques situées à l'ouest de la Catalogne (avril et mai 2008), ainsi qu'à des épisodes de Levant produits par des dépressions méditerranéennes pour ce dernier mois.

Finalement, a titre d'exercice on a appliquée la modèle combinée à la prédiction saisonnière de pluie de toute l'année 2009 (Fig. 8). Dans ce cas on montre la prévision pour périodes trimestrielles. Cet année n'a présenté aucune anomalie important dans la région, et il a été très prochaine a la moyenne climatique. En conséquence, la valeur ajoutée de la méthodologie proposée ici, laquelle peut être très utile pour la prévision de sécheresses, ne peut pas être bien appréciée. Malgré ça on peut voir comme la pluie réelle se trouve dans l'intervalle de précipitation le plus probable. Les exceptions ont été les trimestres FMA, AMJ et JJA

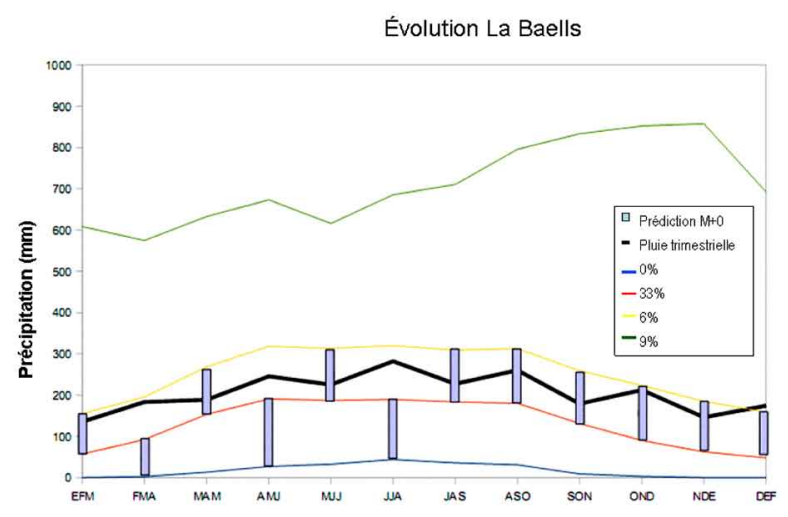

Figure 8. Prédiction de la précipitation Dans le centroide TLL et évolution réelle, pour l'année 2009.

\section{$V \square$ CONCLUSIONS}

Une adaptation de la prédiction saisonnière du modèle ECM4 a été réalisée pour les sources de rivières Ter et Llobregat pour la période août 2001 - décembre 2008. Pour ce faire, nous avons utilisé les registres de précipitations mensuelles couvrant la période 1940 - 2008, et nous avons calculé les séries de pluie de quatre barycentres : celui qui représente le domaine d'étude dans son ensemble (domaine TLL) et trois autres barycentres associés aux sous-bassins alimentant les trois groupes de barrages à étudier (Llosa St. Ponç, Baells et Sau - Susqueda).

L'adaptation à petite échelle du modèle ECM4 a été réalisée au moyen des combinaisons linéaires [8 et 9], ce qui minimise l'erreur quadratique, et a été ajustée à la série du barycentre TLL pour la période de calibrage août 2001 - décembre 2005. A l'intérieur de la prédiction, le premier horizon est celui qui présente le plus grand nombre de réus- 
sites, et pour le reste on obtient toujours des améliorations par rapport aux deux autres modèles. Une validation statistique a été réalisée pour le modèle ECM4, le modèle combiné et le modèle climatique pour la période totale (août 2001 - décembre 2008) et pour la période opérationnelle (janvier 2006 - décembre 2008), au moyen de l'indice BPS et d'un décompte des réussites et des erreurs. Nous arrivons à la conclusion que le modèle combiné améliore les résultats du modèle ECM4 et offre une stabilité en ce qui concerne les réussites. La combinaison permet de retenir pour chaque tercile et pour chaque horizon la prédiction qui est habituellement la meilleure.

Cette prédiction améliorée peut être utilisée dans la gestion des ressources hydriques, et pour ce faire on calcule les seuils de pluie des catégories de la prédiction. Actuellement, les systèmes sur lesquels s'appuie la gestion réalisent une prévision d'évolution des réserves retenues par les barrages, basée sur des méthodes utilisant des données historiques comme informations sur les précipitations. Il est possible de sensiblement améliorer la prévision des réserves en utilisant ces modèles météorologiques saisonniers, en particulier dans le cas d'une période sèche étant donné que ce type de prédictions tient compte des facteurs environnementaux que l'information historique ne peut pas prévoir. En général, on réduit le rang dans lequel l'apport probable peut être situé par rapport au rang obtenu avec des données historiques, mais en outre, dans la majorité des cas, on trouve même la frange la plus probable à l'intérieur de ce même rang, ce qui améliore sensiblement l'information sur l'évolution des ressources hydriques dont le gestionnaire peut disposer. Ceci a été observé pour la majorité des trimestres analysés pour les trois systèmes de réserves étudiés (Sau-Susqueda, La Baells et La Llosa de Cavall-St. Ponç).

\section{VI — REMERCIEMENTS}

Cette contribution a été développée dans le contexte du projet espagnol SOSTAQUA (Projet CÉNIT 2006 approuvée par le CDTI le 14/2/2007).

\section{VII — RÉFÉRENCES ET CITATIONS}

[1] Álvarez J., Potenciano De las Heras A., Villaverde J. J. (2008) - Evolución histórica de sequías en España. Revista de Obras Públicas. 155 (3486) 56-68
[2] Agencia Catalana De L'aigua (2002) - Els recursos hídrics en règim natural a les Conques Internes de Catalunya.

[3] Barnolas M., Atencia A., Llasat M.C., Rigo T. (2008) Characterization of a Mediterranean flash flood event using rain. $A d v$. Geosci. 17 1-7

[4] Barriendos M., Llasat M.C. (2003) - The case of the "Maldá" anomaly in the western mediterranean basin (ad 1760 1800) : an example of a strong climatic variability. Change. 61( 1-2 ) 191-216

[5] Fraedrich K., Leslie L. M. (1987) - Combining predictive schemes in short-term forecasting. Monthly weather review. 116 1640-1644

[6] Fraedrich K., Smith N. R. (1989) - Combining Predictive Schemes in Long-Range Forecasting. Notes and Correspondance, Journal of Climate. 2 291-294

[7] Gibergans-Báguena J., Llasat M. C. (2007) - Improvement of the analog forecasting method by using local thermodynamic data. Application to autumn precipitation in Catalonia. Atmospheric Research. 86 173-193

[8] Lloyd-HughesSAunders M.A. (2002) - A drought climatology for Europe. DOI : 10.1002/joc.846. International Journal of climatology. 22 1571-1592

[9] MARTIN-VidE J., BARRIENDOS M. (1995) - The use of rogation ceremony records in climatic reconstruction : a case study from Catalonia (Spain). Clim. Change. 30 201-221

[10] Мiмам (2000) - Libro blanco del agua. Ministerio de medio ambiente. Secretaría de Estado de Aguas y Costas. Dirección general de Obras Hidráulicas y Calidad de las Aguas.

[11] Miмam (2007) - Planes especiales de sequía. Ministerio de medio ambiente. Secretaría de Estado de Aguas y Costas. Dirección general de Obras Hidráulicas y Calidad de las Aguas. http ://www.mma.es/portal/secciones/acm/aguas_continent_zonas_asoc/ons/planes_sequia_isas.

[12] Olcina J. (2001) - Tipología de sequías en España. Ería. 56 201-227

[13] ZARAGOZA A. (2008) - Mountain meteorology : study of the precipitation in the Pyrenees (in Catalan language). Universitat de Barcelona, Departament d'Astronomia i Meteorologia. 